\title{
Primary Progressive Aphasia: Diagnosis and Contribution of Graphic Markers
}

\author{
Alexandra PLONKA ${ }^{1,2}$, Aurélie MOUTON ${ }^{3}$, Valeria MANERA ${ }^{1,2}$ and Auriane GROS ${ }^{1,2}$ \\ ${ }^{1}$ Université Côte d'Azur, Department of Speech Therapy, France \\ ${ }^{2} \mathrm{CoBTeK}$ (Cognition Behavior Technology) Laboratory, France \\ ${ }^{3}$ Centre Hospitalier Universitaire, Centre Mémoire Ressources et Recherche, France
}

*Corresponding author: Auriane GROS , Université Côte d'Azur, Department of Speech Ther

Received Date: December 17, 2019

Published Date: January 06, 2020

\begin{abstract}
Primary Progressive Aphasia (PPA) is a neurodegenerative disease characterized by a foreground linguistic deficit that extends to other cognitive functions. Structural and cognitive impairments depend of the PPA subtype. Different pathological causes can lead to PPA, such as frontotemporal dementia (FTD) or Alzheimer disease (AD). An early differential diagnosis between the different aetiologies seems crucial to set up an appropriate care but remains difficult to make, according to the several diagnosis changes that can occur. Moreover, non-pharmacological interventions such as speech therapy have proven their usefulness in compensating and maintaining functional communication and the use of graphical parameters have shown a significant handwriting difference between several diseases such as Alzheimer's disease (AD) or Parkinson's disease (PD). In this respect, it would be interesting to take graphic parameters into account within the framework of a classification of parameters characteristic of the neurodegenerative diseases.
\end{abstract}

Keywords: Primary Progressive aphasia; Diagnosis; Graphic markers

Abbreviations: PPA: Primary Progressive Aphasia; PPA-G: Agrammatic subtype of Primary Progressive Aphasia; PPA-S: Semantic Subtype of Primary Progressive Aphasia; PPA-L: Logopenic Subtype of Primary Progressive Aphasia; SD: Semantic Dementia; AD: Alzheimer's Disease; FTD: Fronto Temporal Dementia; PD: Parkinson Disease

\section{Introduction}

Progressive primary aphasia (PPA) brings together a group of neurodegenerative diseases that have the particularity to be characterised by a linguistic deficit which remains isolated before extending to other cognitive functions (such as the executive process, memory, praxis or computation) [1-3]. The starting age of PPA is between 50 and 65 years [4] and survival age is estimated from 10 to 15 years [5]. No aetiological pharmacological intervention treatment is available, however, therapeutic approaches are possible, such as targeted rehabilitation of language. In terms of pharmacology, Bromocriptine [6] and Galantamine [7] were used with small cohorts of patients without conclusive data.

So far, the literature identifies three PPA subtypes: The agrammatic subtype (PPA-G), the semantic subtype (PPA-S) and the logopenic subtype (PPA-L). The semantic subtype has often been assimilated to the concept of semantic dementia (SD) [8-13]. It has been proposed that PPA-S constitutes a purely linguistic variant of the SD [14] or vice versa, that the SD represents an evolved or even more extended form of PPA-S [1]. These phenotypic distinctions have also been studied by those who propose to differentiate the "typical forms" of SD with multi-modal involvement of atypical forms with isolated involvements of the language [15].

\section{Structural impairment and functional impairment}

The aphasic disorders found depends on the type of PPA and the affected structural regions. The expressive side of PPA-G is based on laborious, agrammatic discourses and made of aphasic transformations of phonemic paraphasia type. At the receptive level, understanding depends on the syntactic complexity of the sentence $[3,16,17]$. The lesion is located in the lower frontal cortex, 
in the Broca region and the anterior parts of the insula [10,18-20]. Other authors have also shown parietal and temporal lesions [20].

PPA-L is based, at the expressive level, on a lack of the word that alters the fluidity of the language. Understanding is preserved at the beginning, but can be altered in illness evolution, due to disorders of the phonological loop. Understanding is dependent on the length of sentences [10-11]. It is characterized by lesions of the posterior external temporary cortex and the parietal cortex with Wernicke's air [10,20,21].

PPA-S expressive aspects are characterized by semantic paraphasias and receptive aspects are characterized by impairments of word comprehension in the oral or written mode $[14,22]$. PPA- S is characterized by an involvement of the anterior temporal cortex $[10,23,24]$ and the inferior and middle temporal cortex [25]. A fiber-tracking method (DTI) [21] on a small sample $(\mathrm{N}=5)$ also reported an infiltration of several connecting beams passing through the temporal lobe. PPA mixed are characterised by several symptoms of the three forms previously presented.

\section{Diagnosis and evolution of APP}

APP can be due to different pathological causes, most commonly frontotemporal dementia (FTD) or Alzheimer disease (AD). The early differential diagnosis between the different aetiologies seems crucial as it could induce the avoidance of inappropriate use of anticholinesterase inhibitors (AchI). In addition, some APP phenotype should lead to inappropriate AchI use. AchI seem not appropriate to the FTD physiopathology as prominent cholinergic deficit has been identified only in AD [26]. Atypical AD is suspected facing some PPA phenotypes and along their evolution. Cholinergic treatment may be beneficial for an atypical AD but is suspected to worsen behavioral symptoms of fronto temporal dementia (FTD). The PPA diagnosis is made when the three following criteria are met: there is a progressive aphasic disorder that increases distortions of word usage or comprehension; the language impairment constitutes the most salient deficit and causes most of the daily living activities impairment during the initial stages of illness; and the underlying disease is considered neurodegenerative [1]. The neurodegenerative diagnosis trajectory is still debated as several diagnosis changes occur during the course of this disease due to phenotype evolution from isolated language alterations to global cognitive impairment with multiple neuropsychiatric symptoms 7. Even if the PPA duration is estimated at about 6 years, language symptoms could stay the only set of signs for as many as 10-14 years. After few years, in addition to psychiatric and neurologic symptoms, deficits in new cognitive domains appear but the language dysfunction seems to remain the most salient feature and to be impaired the most quickly throughout the degeneration process [8,27]. PPA-L is considered as an atypical phenotype of Alzheimer's disease (AD) [28]. PPA then evolves to a more generalized cognitive impairment and is so called PPA-plus. PPA patients could exaggerate their acalculia and ideomotor/visuoconstructive apraxia that even exists but stayed non-prominent hitherto. Several neuropsychiatric symptoms could also emerge [29]. In a longitudinal follow-up study with clinicopathological analysis, 54\% of the cohort developed a second or third syndrome other than PPA (FTD, progressive supranuclear palsy, or corticobasal degeneration) [9]. Neurodegenerative diagnosis underlying PPA still a major outcome as this phenotype is evolving. The underlying physiopathology is not clear as several nonspecific anomalies have been identified and non-highly specific clinicopathological correlation is reported.

\section{Contribution of graphic markers in the early and differential diagnosis of Primary Progressive Aphasia}

Although the literature on biomarkers of neurodegenerative pathologies and brain damage is abundant, research on the parameters of daily life activities associated with global and fine motor functions remains scarcer. Nevertheless, their consideration seems essential for two major points:

- The precise analysis of the changes observed at the preclinical stages will allow a better understanding and a more global vision compared to other neurocognitive disorders.

These analyzes could allow the development of noninvasive and inexpensive tools to support conventional, invasive and more costly techniques (lumbar puncture or PET Scan).

Markers of this type appear to be particularly relevant in differential diagnosis because the biomarkers currently used have been criticized for their lack of specificity [30], in particular because individuals with biomarkers in vivo may never present with clinical symptoms [31]. Considering behavioral parameters in a differential context with other pathologies could thus bring more reliability in the diagnosis. As these ecological methods can now be recorded by high quality and generally inexpensive sensors (digital tablets, digital voice recorders) with high temporal and spatial resolution, various experts have encouraged their use for diagnosis [32-34]. Regarding graphic markers, several studies have revealed that they are affected early in people with moderate to severe Alzheimer's disease [35]. Studies have also shown that motor activity reveals language-related characteristics, due to the involvement of motor areas of the brain in writing [36], and that even mild disorders can be detected by means of motor parameters (reduction in written pressure) during language production tasks. Handwriting requires the implementation of cognitive processes related to language as well as planning, coordination and motor performance. It has thus been shown that people with cognitive decline overall have a lower writing speed and pressure with a longer writing time, and this especially when analyzing cursive loops [37]. The performances of handwriting know therefore a significant change which it would be interesting to take into account within the framework of a classification of parameters characteristic of the neurodegenerative diseases such as Alzheimer's disease (AD), Parkinson's disease (PD)) or the PPA [38].

\section{Conclusion}

To date, there is no pharmacological treatment modifying or delaying PPA. However, non-pharmacological interventions, such as speech therapy [39] have proven to be useful in compensating 
for and maintaining functional communication. Early diagnosis of PPA is therefore crucial because it increases the possibilities of appropriate clinical interventions, coordinated care plans, symptom management, improvement of patient safety, but also the possibility of reducing health costs and the period before the institutionalization of affected persons. Nevertheless, the diagnosis of APP is a major challenge in clinical practice because this phenotype is complex and constantly evolving. Language symptoms being the earliest and most prominent signs at the beginning of the pathology, graphic writing markers, little studied until now like the voice, could constitute ecological indices of great interest for the early diagnosis of PPA.

\section{Acknowledgement}

None.

\section{Conflict of Interest}

No conflict of interest.

\section{References}

1. MM Mesulam (2001) Primary progressive aphasia. Ann Neurol 49(4): 425-432.

2. MM Mesulam (2003) Primary Progressive Aphasia-A Language-Based Dementia. New England Journal of Medicine 349(16): 1535-1542.

3. M Grossman, S Ash (2004) Primary Progressive Aphasia: A Review. Neurocase 10(1): 3-18.

4. E Ratnavalli, C Brayne, K Dawson, JR Hodges (2002) The prevalence of frontotemporal dementia. Neurology 58(11): 1615-1621.

5. E Le Rhun, F Richard, F Pasquier (2005) Natural history of primary progressive aphasia. Neurology 65(6): 887-891.

6. DA Reed, NA Johnson, C Thompson, S Weintraub, MM Mesulam (2004) A clinical trial of bromocriptine for treatment of primary progressive aphasia. Annals of Neurology 56(5): 750-750.

7. Kertesz A, Morlog D, Light M, Blair M, Davidson W, et al. (2008) Galantamine in Frontotemporal Dementia and Primary Progressive Aphasia. Dementia and Geriatric Cognitive Disorders 25(2): 178-185.

8. S Weintraub, NP Rubin, MM Mesulam (1990) Primary progressive aphasia. Longitudinal course, neuropsychological profile, and language features. Arch Neurol 47(12): 1329-1335.

9. A Kertesz, W Davidson, P Mccabe, K Takagi, D Munoz (2003) Primary progressive aphasia: Diagnosis, varieties, evolution. Journal of the International Neuropsychological Society 9(5): 710-719.

10. M. L. Gorno-Tempini et al., (2004) Cognition and anatomy in three variants of primary progressive aphasia. Annals of Neurology 55(3): 335-346.

11. M. L. Gorno-Tempini et al., (2008) The logopenic/phonological variant of primary progressive aphasia. Neurology 71(16): 1227-1234.

12. JA Knibb, JR Hodges (2005) Semantic dementia and primary progressive aphasia: a problem of categorization? Alzheimer Dis Assoc Disord 19(1): S7-14.

13. S Amici, ML Gorno-Tempini, JM Ogar, NF Dronkers, BL Miller (2006) An overview on Primary Progressive Aphasia and its variants. Behav Neurol 17(2): 77-87.

14. Adlam AL, Patterson K, Rogers TT, Nestor PJ, Salmond CH, et al. (2006) Semantic dementia and fluent primary progressive aphasia: two sides of the same coin? Brain 129(11): 3066-3080.

15. Moreaud O, Belliard S, Snowden J, Auriacombe S, Basaglia-Pappas S, et al. (2008) Démence sémantique: réflexions d'un groupe de travail pour des critères de diagnostic en français et la constitution d'une cohorte de patients. Revue Neurologique 164(4): 343-353.
16. Grossman M, Mickanin J, Onishi K, Hughes E, D’Esposito M, et al. (1996) Progressive Nonfluent Aphasia: Language, Cognitive, and PET Measures Contrasted with Probable Alzheimer's Disease. Journal of Cognitive Neuroscience 8(2): 135-154.

17. JA Knibb, AM Woollams, JR Hodges, K. Patterson (2009) Making sense of progressive non-fluent aphasia: an analysis of conversational speech. Brain 132(10): 2734-2746.

18. Josephs KA, Duffy JR, Strand EA, Whitwell JL, Layton KF, et al. (2006) Clinicopathological and imaging correlates of progressive aphasia and apraxia of speech. Brain 129(6): 1385-1398.

19. Rohrer JD, Warren JD, Modat M, Ridgway GR, Douiri A, et al, (2009) Patterns of cortical thinning in the language variants of frontotemporal lobar degeneration. Neurology 72(18): 1562-1569.

20. Rohrer JD, Ridgway GR, Crutch SJ, Hailstone J, Goll JC, et al. (2010) Progressive logopenic/phonological aphasia: Erosion of the language network. Neuro Image 49(1): 984-993.

21. Agosta F, Henry RG, Migliaccio R, Neuhaus J, Miller BL, et al. (2010) Language networks in semantic dementia. Brain 133(1): 286-299.

22. JR Hodges, K Patterson, S Oxbury, E Funnell (1992) Semantic dementia. Progressive fluent aphasia with temporal lobe atrophy. Brain 115(Pt 6): 1783-1806.

23. CJ Mummery, K Patterson, CJ Price, J Ashburner, RS Frackowiak, et al. (2000) A voxel-based morphometry study of semantic dementia: relationship between temporal lobe atrophy and semantic memory. Ann Neurol 47: 36-45.

24. Rosen HJ, Perry RJ, Murphy J, Kramer JH, Mychack P, et al. (2002) Emotion comprehension in the temporal variant of frontotemporal dementia. Brain 125(10): 2286-2295.

25. P Garrard, JR Hodges (2000) Semantic dementia: clinical, radiological and pathological perspectives. Journal of Neurology 247(6): 409-422.

26. GA Kerchner, MC Tartaglia, AL Boxer (2011) Abhorring the vacuum: use of Alzheimer's disease medications in frontotemporal dementia. Expert Review of Neurotherapeutics 11(5): 709-717.

27. M Mesulam (2013) Primary progressive aphasia: A dementia of the language network. Dement Neuropsychol 7(1): 2-9.

28. B Dubois et al., (2010) Revising the definition of Alzheimer's disease: a new lexicon. Lancet Neurol 9(11): 1118-1127.

29. Matias-Guiu JA, Díaz-Álvarez J, Ayala JL, Risco-Martín JL, Moreno-Ramos T, et al. (2018) Clustering Analysis of FDG-PET Imaging in Primary Progressive Aphasia. Front Aging Neurosci 10: 230.

30. MM Mesulam, S Weintraub, EJ Rogalski, C Wieneke, C Geula, et al. (2014) Asymmetry and heterogeneity of Alzheimer's and frontotemporal pathology in primary progressive aphasia. Brain 137(Pt 4): 1176-1192.

31. Nelson PT, Alafuzoff I, Bigio EH, Bouras C, Braak H, et al. (2012) Correlation of Alzheimer Disease Neuropathologic Changes with Cognitive Status: A Review of the Literature. Journal of Neuropathology \& Experimental Neurology 71(5): 362-381.

32. H Takao, N Hayashi, K Ohtomo (2012) A longitudinal study of brain volume changes in normal aging. European Journal of Radiology 81(10): 2801-2804.

33. MM Mielke et al. (2013) Assessing the Temporal Relationship Between Cognition and Gait: Slow Gait Predicts Cognitive Decline in the Mayo Clinic Study of Aging. The Journals of Gerontology Series A: Biological Sciences and Medical Sciences. 68(8): 929-937.

34. Auriane Gros, David Bensamoun, Valeria Manera, Roxane Fabre, AnneMarie Zacconi-Cauvin, et al. (2016) Recommendations for the Use of ICT in Elderly Populations with Affective Disorders. Frontiers in Aging Neuroscience 8.

35. O Afonso, CJ Álvarez, C Martínez, F Cuetos (2019) Writing difficulties in Alzheimer's disease and mild cognitive impairment. Read Writ 32(1): 217-233.

36. Tatjana A Nazir, Lianna Hrycyk, Quentin Moreau, Victor Frak, Anne Cheylus, et al. (2017) A simple technique to study embodied language processes: the grip force sensor. Behav Res 49(1): 61-73. 
37. C Kahindo, MA El-Yacoubi, S Garcia Salicetti, AS Rigaud, V CristanchoLacroix (2018) Characterizing Early-Stage Alzheimer Through Spatiotemporal Dynamics of Handwriting. IEEE Signal Processing Letters 25(8): 1136-1140.

38. A Gros, A Plonka, V Manera (2019) Graphic Markers: Towards an Early Diagnosis of Primary Progressive Aphasia. Alzheimer's \& Dementia 15(7): P351-P352.
39. S Routhier, K Gravel-Laflamme, J Macoir (2013) Non-pharmacological therapies for language deficits in the agrammatic and logopenic variants of primary progressive aphasia: a literature review. Gériatrie et Psychologie Neuropsychiatrie du Viellissement 11(1): 87-97. 